\title{
Using Murray's law to design artificial vascular microfluidic networks
}

\author{
R. W. Barber ${ }^{1}$, K. Cieślicki ${ }^{2}$ \& D. R. Emerson ${ }^{1}$ \\ ${ }^{I}$ Centre for Microfluidics and Microsystems Modelling, \\ CCLRC Daresbury Laboratory, Warrington, UK \\ ${ }^{2}$ Institute of Automatic Control and Robotics, \\ Warsaw University of Technology, Poland
}

\begin{abstract}
A generalised version of Murray's law has been derived for the design of microfluidic manifolds and hierarchical fluid distribution systems. Murray's law was original obtained from a study of mammalian cardiovascular systems and describes the optimum conditions governing the ratio of diameters of the vessels in a branching vascular network. The optimum geometrical relationship, which is now known as Murray's law, states that the cube of the diameter of the parent vessel must equal the sum of the cubes of the daughter vessels. When the parent/daughter branches obey Murray's law, the system obeys the principle of minimum work. Furthermore, if the network consists of symmetric bifurcations, an important consequence of Murray's law is that the tangential shear stress at the wall remains constant throughout the vascular system. In the present paper, we generalise this important hydrodynamic principle and provide a biomimetic design rule for microfluidic systems composed of arbitrary cross-sections. In particular, the paper focuses on the design of constant-depth rectangular- and trapezoidal-sectioned microfluidic manifolds that are often used in lab-on-a-chip systems. To validate the biomimetic design principles, a comprehensive series of computational fluid dynamic simulations have been performed. Keywords: biomimetic, microfluidic, vascular, manifold, lab-on-a-chip.
\end{abstract}

\section{Introduction}

It is evident that nature has perfected techniques and solutions that are often considered to be optimal. Understanding and extracting these "natural" design 
strategies has opened up a whole new field of research known as biomimetics. Designs formulated using biomimetic principles range from novel surface treatments that mimic physiological processes to geometrical optimisation that improves the performance of a system.

One research area where biomimetic principles could play an important role is in the design of microfluidic and lab-on-a-chip systems. In recent years, microfluidic devices have been increasingly used in a range of chemical, biochemical and life-science applications. Miniaturisation offers many potential benefits including faster mixing and reaction times, increased chemical yields, and faster throughput rates for chemical assays. In addition, the small length scales of microfluidic systems offer the prospect of developing portable detection systems for point-of-care clinical diagnostics. However, little research is currently available concerning the optimisation of channel dimensions to ensure the most efficient flow through the device.

The present paper shows how biomimetic principles based on the laws that govern biological vascular trees could be used to design artificial microfluidic distribution systems. The study focuses specifically on microfluidic manifolds composed of constant-depth rectangular- or trapezoidal-sectioned channels that can readily be fabricated using standard micro-fabrication techniques such as photolithography and wet or dry etching. Furthermore, by carefully selecting a branching parameter governing each bifurcation, it is shown that it is possible to introduce a prescribed element of control into the flow behaviour in the system.

\section{Theoretical basis of Murray's law}

The geometrical configurations of vessels found in mammalian cardiovascular and respiratory systems have evolved, through natural selection, to an optimum arrangement that minimises the amount of biological work required to operate and maintain the system. The most distinctive feature of biological distribution systems is their hierarchical structure and the successive division of vessels which become smaller, both in length and diameter (as illustrated schematically in Fig. 1). The relationship between the diameter of the parent and daughter vessels was first derived by Murray [1] using the principle of minimum work. Murray found that the optimum relation between the diameter of the parent vessel $\left(d_{0}\right)$ and the two daughter branches $\left(d_{1 a}\right.$ and $\left.d_{1 b}\right)$ can written as

$$
d_{0}^{3}=d_{1 a}^{3}+d_{1 b}^{3} .
$$

This expression is nowadays known as Murray's law but it is sometimes referred to as "the third power law". For a symmetric bifurcation where $d_{1 a}=d_{1 b}$, eqn. (1) reduces to

$$
d_{0}^{3}=2 d_{1}^{3}
$$

By making the assumption that the flow is fully-developed and ignoring the energy losses at each bifurcation, it is possible to obtain relationships between vessel diameters, average velocity, wall shear stress, flow resistance, pressure, and residence time for each consecutive generation of the vascular system. 
The work of Murray was overlooked for almost half a century, but recently biological scaling laws are beginning to receive more attention. However, with the exception of the brief study by Lim et al. [2], there appears to be little application of Murray's law to the design of man-made structures and, in particular, to the design of microfluidic channels and manifolds.

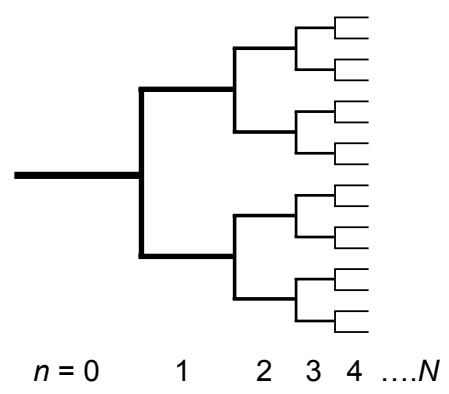

(a)

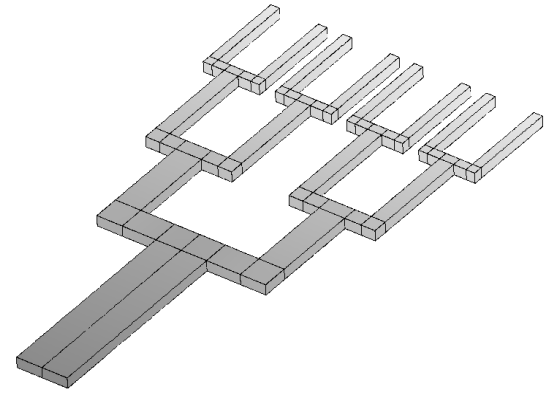

(b)

Figure 1: (a) Schematic representation of a symmetric bifurcating network of channels; (b) Layout of a typical constant-depth rectangular microfluidic manifold considered in the validation study.

\subsection{Generalisation of Murray's law}

As demonstrated by Cieślicki [3], Murray's law can be generalised if the change in diameter of each consecutive generation can be represented by a branching parameter, $X$ :

$$
X=\frac{d_{0}^{3}}{2 d_{1}^{3}} .
$$

For $X=1$, the parent/daughter branches obey the principle of minimum work. However, the branching parameter does not have to be unity and the generalised case of $X \neq 1$ can be used to design microfluidic manifolds with specific properties e.g. shear stress distributions or residence times. Assuming that the branching parameter is held constant throughout the hierarchical network, then the segment diameter of the $n^{\text {th }}$ generation can be written as

$$
d_{n}=\frac{d_{0}}{(2 X)^{n / 3}} .
$$

Fig. 2(a) shows graphically how eqn. (4) behaves for a range of $X$ values. It can be seen that the diameters systematically diminish when the branching parameter is greater than 0.5 . For $X=0.5$, the diameter at each generation is constant, whereas when $X<0.5$, each diameter will systematically increase. For Murray's law, $(X=1)$, the damping factor is equal to $2^{-1 / 3}$. This implies that the diameter of a segment will be halved after three successive generations. 
248 Design and Nature III: Comparing Design in Nature with Science and Engineering

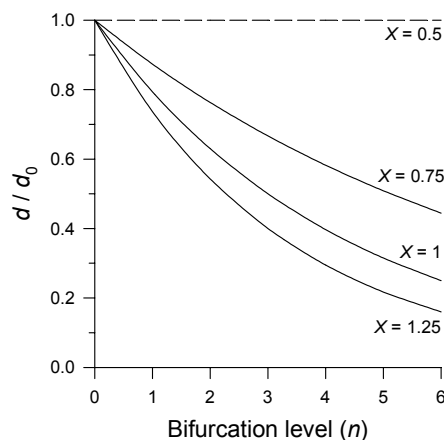

(a)

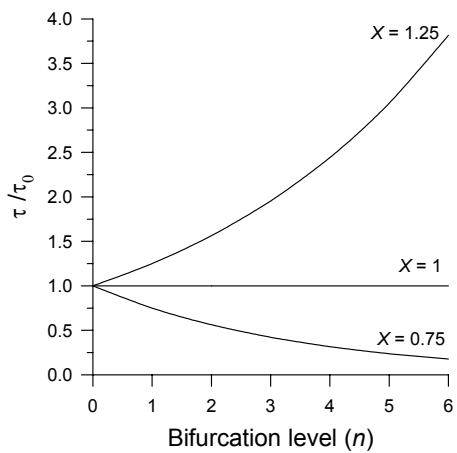

(c)

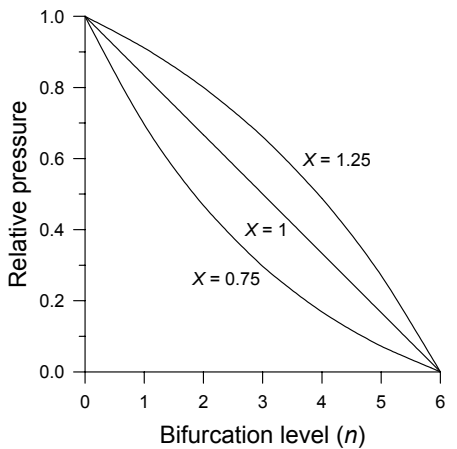

(e)

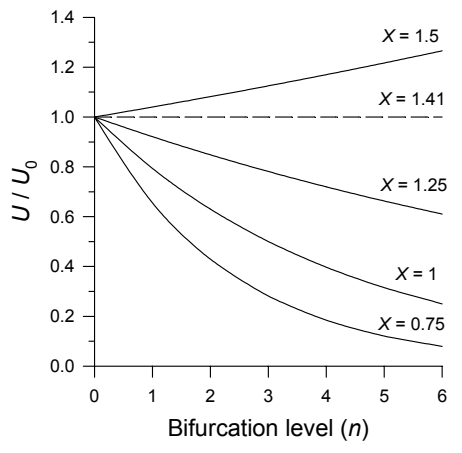

(b)

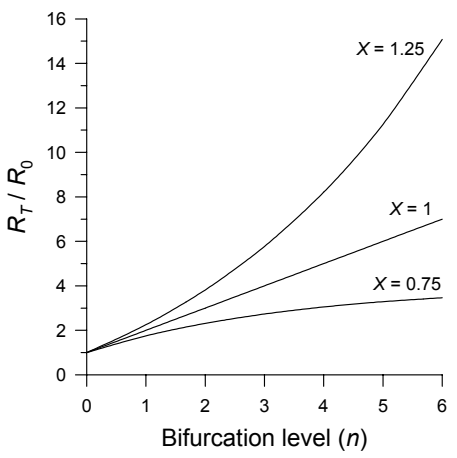

(d)

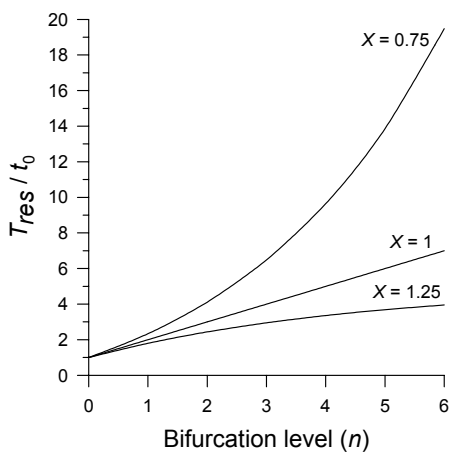

(f)

Figure 2: Normalised segment diameter (a), average flow velocity (b), wall shear stress (c), flow resistance (d), pressure distribution (e) and residence time (f) as a function of bifurcation level, $n$, in a vascular system that obeys the generalised form of Murray's law. 
For a symmetric system, the volumetric flow rate halves at each bifurcation i.e. $Q_{n}=2^{-n} Q_{0}$. Using eqn. (4), the mean flow velocity, $\bar{U}_{n}$, in each generation can be shown to be

$$
\bar{U}_{n}=\bar{U}_{0}\left(\frac{1}{2} X^{2}\right)^{n / 3} .
$$

Fig. 2(b) shows the predicted velocity behaviour throughout the manifold as the branching parameter $X$ is varied. It can be seen that if $X<\sqrt{2}$, the average velocity will decrease in each generation.

The shear stress acting on the wall of a circular pipe in a fully-developed laminar flow can be written as follows:

$$
\tau=\frac{8 \mu \bar{U}}{d},
$$

where $\mu$ is the fluid viscosity. Substituting eqns. (4) and (5) into (6) gives

$$
\tau_{n}=\tau_{0} X^{n},
$$

where $\tau_{0}$ is the wall shear stress in the inlet channel $(n=0)$. Eqn. (7) is plotted in Fig. 2(c) and clearly illustrates that if Murray's law is obeyed $(X=1)$, the magnitude of the wall shear stress remains the same at every point in the branching hierarchy. However, by changing the value of $X$, it is possible to introduce an element of control into the shear stress distribution.

The hydraulic resistance of a single segment is defined as $\Delta P / Q$ and can be obtained from the Hagen-Poiseuille pipe friction formula. Furthermore, if the length of an individual segment is assumed to be proportional to its diameter $\left(L_{n} \propto d_{n}\right)$, as frequently observed in biological systems [4], the resistance of a single segment in the $n^{\text {th }}$ generation can written as

$$
R_{n} \propto d_{n}^{-3} .
$$

Using eqns. (4) and (8) allows the change of hydraulic resistance of consecutive generations to be related to the branching parameter $X$ :

$$
R_{n}=R_{0}(2 X)^{n} .
$$

The total resistance, $R_{T}$, of a bifurcating vascular tree can thus be written as

$$
R_{T}=R_{0}+\frac{R_{1}}{2}+\frac{R_{2}}{4}+\frac{R_{3}}{8}+\ldots+\frac{R_{n}}{2^{n}}+\ldots+\frac{R_{N}}{2^{N}}=R_{0} \sum_{i=0}^{N} X^{i}=R_{0} \frac{X^{N+1}-1}{X-1} .
$$

For Murray's law $(X=1)$, eqn. (10) reduces to $R_{T}=(N+1) R_{0}$. Examples of the total resistance of the network are shown in Fig. 2(d) for several values of $X$. When Murray's law is obeyed, the resistance of each generation is identical, so the total resistance to the flow increases linearly with the number of generations. For $X>1$ the resistance of subsequent generations increases rapidly. However, for $X<1$, the resistance of subsequent generations decreases and the total resistance will tend to a constant value of $R_{T}=R_{0} /(1-X)$ as $N \rightarrow \infty$. 
Using the analogy between pipe friction and electrical resistance allows the pressure to be determined throughout the network. It can be shown that

$$
\frac{p_{n}-p_{\text {out }}}{p_{\text {in }}-p_{\text {out }}}=\frac{\sum_{i=n}^{N} X^{i}}{\sum_{i=0}^{N} X^{i}}=\left(\frac{X^{N+1}-X^{n}}{X^{N+1}-1}\right),
$$

where $p_{n}$ is the pressure at the entrance to the $n^{\text {th }}$ generation and $p_{\text {in }}$ and $p_{\text {out }}$ are the pressures at the inlet and outlet of a branching network with $N$ bifurcation levels. Fig. 2(e) shows the normalised pressure distribution for a vascular system with 6 generations and several values of the branching parameter. For $X<1$, the most significant loss of pressure occurs in the inlet channel $(n=0)$ with the pressure drop gradually diminishing at each successive generation, leading to a concave distribution. Conversely, for $X>1$, the pressure drop becomes most significant at the outlet of the hierarchical tree, giving a convex profile. When $X=1$, the pressure loss along each successive generation is constant, leading to a linear pressure distribution along the vascular structure.

If the length of an individual segment is again considered to be proportional to its diameter (i.e. $L_{n}=k d_{n}$ where $k$ is a constant), then the average residence time for a single segment in the $n^{\text {th }}$ generation, $t_{n}$, can be written as

$$
t_{n}=\frac{L_{n}}{\bar{U}_{n}}=\frac{k d_{n}}{\bar{U}_{n}}=\frac{k d_{0}}{\bar{U}_{0} X^{n}}=\frac{L_{0}}{\bar{U}_{0} X^{n}}=\frac{t_{0}}{X^{n}} .
$$

The total residence time, $T_{\text {res }}$, for the entire vascular tree with $N$ bifurcation levels will then be

$$
T_{\text {res }}=t_{0} \sum_{i=0}^{N} \frac{1}{X^{i}}=t_{0} \frac{X^{N+1}-1}{X^{N+1}-X^{N}} .
$$

Typical residence time profiles are shown in Fig. 2(f) for different values of the branching parameter $X$. For $X<1$, the time to flow through each successive generation increases with the number of generations. Conversely, for $X>1$, the residence time decreases at each successive generation and the total residence time converges to a normalised value of $X /(X-1)$.

\subsection{Extension of Murray's law to non-circular ducts}

The extension of biomimetic principles to non-circular ducts has recently been demonstrated by Emerson et al. [5] who proposed that the design rule should be defined using an analogous expression to eqn. (7) but based on the mean value of the tangential shear stress in each segment, i.e.

$$
\bar{\tau}_{n}=\bar{\tau}_{0} X^{n} .
$$


The mean wall shear stress can be related to the Fanning friction factor, $f$, which in turn can be expressed in terms of the Poiseuille and Reynolds numbers [6]:

$$
\bar{\tau}=\frac{1}{2} \rho \bar{U}^{2} f=\frac{1}{2} \rho \bar{U}^{2} \frac{\mathrm{Po}}{\mathrm{Re}}=\frac{\mu \bar{U} \mathrm{Po}}{2 D_{\mathrm{h}}},
$$

where $D_{\mathrm{h}}=4 \times$ area/wetted perimeter is the hydraulic diameter of the crosssection and $\bar{U}$ is the mean flow velocity. Substituting eqn. (15) into (14) gives a generalised biomimetic principle that can be applied to all channel shapes irrespective of the cross-sectional geometry:

$$
\frac{\bar{U}_{\mathrm{n}} \mathrm{Po}_{\mathrm{n}}}{D_{\mathrm{h} n}}=\frac{\bar{U}_{0} \mathrm{Po}_{0}}{D_{\mathrm{h} 0}} X^{n}
$$

The only practical limitation on the use of eqn. (16) as a biomimetic design rule is the requirement to know the hydraulic diameter and the Poiseuille number of the cross-section. As an aside, for vascular systems composed of circular pipes, the Poiseuille number is identical in each generation $(\mathrm{Po}=16)$ and the hydraulic diameter, $D_{\mathrm{h}}$, is equal to the diameter of the section, $d$. Under these conditions, eqn. (16) reduces to eqn. (5).

\subsection{Application to constant-depth biomimetic networks}

The present study focuses on microfluidic manifolds composed of constant-depth rectangular- or trapezoidal-sectioned channels since these geometries can readily be fabricated using conventional micro-fabrication techniques such as photolithography and wet or dry etching.

For a system composed of constant-depth rectangular channels, as typically encountered in dry etching techniques, the aspect ratio of the $n^{\text {th }}$ generation can be defined as $\alpha_{n}=d / w_{n}$ where $d$ is the depth and $w_{n}$ is the width of the channel. After some algebraic manipulation, it can be shown that the biomimetic design rule defined in eqn. (16) can be written as a function of the aspect ratio:

$$
\alpha_{n}\left(1+\alpha_{n}\right) \operatorname{Po}\left(\alpha_{n}^{*}\right)=(2 X)^{n} \alpha_{0}\left(1+\alpha_{0}\right) \operatorname{Po}\left(\alpha_{0}^{*}\right) .
$$

The Poiseuille number for a rectangular cross-section can be determined analytically [6] as follows:

$$
\operatorname{Po}\left(\alpha_{n}^{*}\right)=24\left[1-\frac{192}{\pi^{5}} \frac{1}{\alpha_{n}^{*}} \sum_{i=1,3,5 \ldots}^{\infty} \frac{1}{i^{5}} \tanh \left(\frac{i \pi \alpha_{n}^{*}}{2}\right)\right]^{-1}\left(1+\frac{1}{\alpha_{n}^{*}}\right)^{-2} .
$$

Eqn. (18) is valid when $\alpha_{n}^{*} \leq 1$, which requires the width to be greater than the depth. To obtain the value of the Poiseuille number when the width is less than the depth $\left(\alpha_{n}>1\right)$, it is necessary to set $\alpha_{n}^{*}=w_{n} / d$ but it is important to note that $\alpha_{n}$ in eqn. (17) remains as previously defined (i.e. $\alpha_{n}=d / w_{n}$ ). Any appropriate method for finding the root, $\alpha_{n}$, can be used and in the present study a simple bisection method has been found to be reliable. 
Another important fabrication technique for constructing microfluidic channels involves anisotropically etching [100] silicon wafers using $\mathrm{KOH}$. This results in a trapezoidal section with an angle of $\tan ^{-1}(\sqrt{2})=54.74^{\circ}$. It can be shown that the biomimetic design rule for $\mathrm{KOH}$ etched silicon channels can be written as

$$
\frac{\sqrt{2} \gamma_{n}+(\sqrt{3}-1) \gamma_{n}^{2}}{\left(\sqrt{2}-\gamma_{n}\right)^{2}} \operatorname{Po}\left(\gamma_{n}\right)=(2 X)^{n} \frac{\sqrt{2} \gamma_{0}+(\sqrt{3}-1) \gamma_{0}^{2}}{\left(\sqrt{2}-\gamma_{0}\right)^{2}} \operatorname{Po}\left(\gamma_{0}\right) \text {, }
$$

where $\gamma=d / a$ is the aspect ratio of the channel, defined in terms of the upper width, $a$. For many practical channel geometries, including trapezoidal channels, the Poiseuille number cannot be obtained analytically. However, Morini [7] has shown that the Poiseuille number for $\mathrm{KOH}$-etched [100] silicon channels can be determined from a $5^{\text {th }}$ order polynomial as follows:

$$
\operatorname{Po}\left(\gamma_{n}\right)=24\left[1-b_{1} \gamma_{n}+b_{2} \gamma_{n}^{2}-b_{3} \gamma_{n}^{3}+b_{4} \gamma_{n}^{4}-b_{5} \gamma_{n}^{5}\right],
$$

where the coefficients have the values of $b_{1}=1.7611, b_{2}=2.6780, b_{3}=4.9342$, $b_{4}=10.0883$, and $b_{5}=7.4496$, respectively.

\section{Numerical validation}

The generalised biomimetic design rules for constant-depth channels have been validated by conducting a comprehensive series of computational fluid dynamic simulations on a range of rectangular- and trapezoidal-sectioned manifolds. The networks were restricted to four generations $(n=0,1,2,3)$ and the channels were assumed to be $125 \mu \mathrm{m}$ deep. For the rectangular channels, the initial aspect ratio was assumed to be either $2: 1\left(\alpha_{0}=0.5\right)$ or $5: 1\left(\alpha_{0}=0.2\right)$, while the initial aspect ratio for the trapezoidal section was taken to be $8: 1\left(\gamma_{0}=0.125\right)$. Table 1 presents the dimensions of the channels used in the numerical study. The channel widths were obtained by solving either eqn. (17) for $\alpha_{n}$ or eqn. (19) for $\gamma_{n}$.

Table 1: $\quad$ Channel dimensions employed in the numerical study.

\begin{tabular}{|c|c|c|c|c|c|}
\hline \multirow{2}{*}{$\begin{array}{c}\text { Bifurcation } \\
\text { level, } n\end{array}$} & \multicolumn{5}{|c|}{ Channel widths $(\mu \mathrm{m})$} \\
\cline { 2 - 6 } & $X=0.75$ & $X=1.0$ & $X=1.25$ & $X=1.0$ & Trapezoidal \\
\cline { 2 - 6 } & 250.0 & 250.0 & 250.0 & 625.0 & 1000.0 \\
\hline 0 & 177.7 & 143.3 & 123.0 & 312.9 & 536.7 \\
\hline 1 & 132.0 & 91.8 & 71.4 & 171.5 & 323.9 \\
\hline 2 & 101.7 & 62.5 & 44.2 & 106.3 & 230.1 \\
\hline 3 & &
\end{tabular}


The numerical simulations were conducted using the commercial computational fluid dynamics software package, CFD-ACE+ (ESI CFD, Huntsville, USA [8]). Fig. 3 shows the predicted normalised wall shear stress distribution in a rectangular microfluidic manifold and illustrates the progressive decrease in shear stress through the vascular system when $X<1$. The effect of varying the branching parameter is further demonstrated in Fig. 4 which shows the normalised shear stress distribution and flow resistance for a range of channel aspect ratios and branching parameters. The theoretical and numerical predictions are in very good agreement, demonstrating the applicability of the proposed biomimetic design principle.

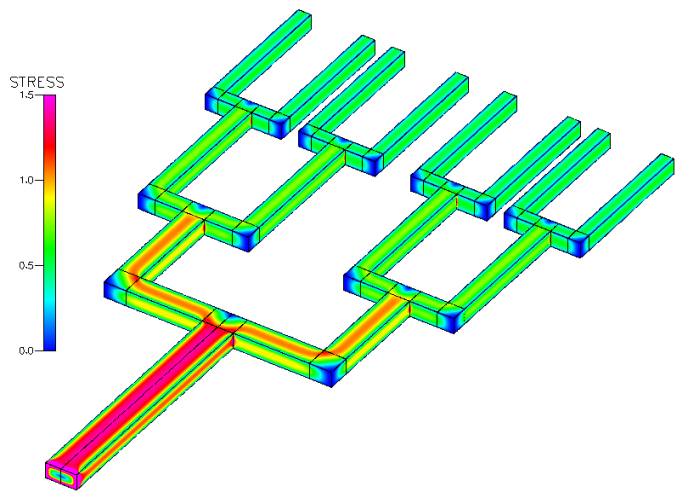

Figure 3: Predicted normalised wall shear stress distribution $\left(\bar{\tau}_{n} / \bar{\tau}_{0}\right)$ in a rectangular microfluidic manifold with a branching parameter of $X=0.75$ and an initial aspect ratio of $\alpha_{0}=0.5$.

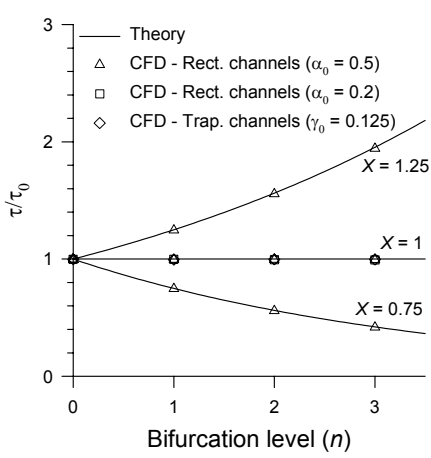

(a)

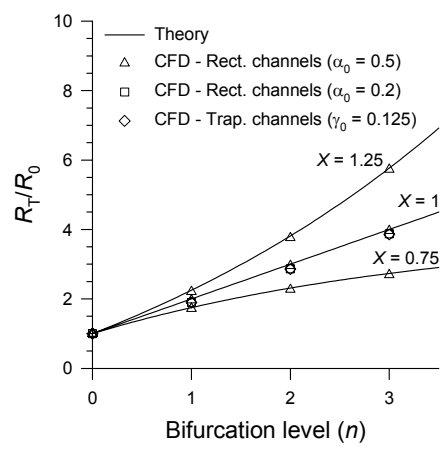

(b)

Figure 4: Normalised wall shear stress distribution (a) and flow resistance (b) in constant-depth rectangular and trapezoidal vascular systems that obey the generalised form of Murray's law. Comparison between theoretical predictions (lines) and CFD results (symbols). 


\section{Conclusions}

Murray's law was originally developed for cardiovascular systems composed of multi-diameter circular pipes. For symmetric bifurcating systems, an important consequence of Murray's law is that the tangential shear stress at the wall remains constant throughout the vascular network. In the present paper, this important biomimetic principle has been generalised so that it is applicable to microfluidic networks composed of channels of arbitrary cross-section. The paper focuses specifically on the design of constant-depth rectangular- and trapezoidal-sectioned microfluidic manifolds that are often used in lab-on-a-chip systems. By carefully selecting a branching parameter governing the change in channel dimension at each bifurcation, it is shown that it is possible to introduce an element of flow control into the artificial vascular network.

\section{Acknowledgements}

The authors would like to acknowledge partial support from the Framework VI PATENT DfMM Network of Excellence (contract no. 507255). Additional support was provided by the UK Engineering and Physical Sciences Research Council (EPSRC) under the auspices of Collaborative Computational Project 12.

\section{References}

[1] Murray, C.D., The physiological principle of minimum work: I. The vascular system and the cost of blood volume, Proc. Natl. Acad. Sci. USA, 12, pp. 207-214, 1926.

[2] Lim, D., Kamotani, Y., Cho, B., Mazumder, J. \& Takayama, S., Fabrication of microfluidic mixers and artificial vasculatures using a high-brightness diode-pumped Nd:YAG laser direct write method, Lab Chip, 3, pp. 318-323, 2003.

[3] Cieślicki, K., Resistance to the blood flow of a vascular tree - a model study, Polish Journal of Medical Physics and Engineering, 5, pp. 161-172, 1999.

[4] West, B.J., Fractal Physiology and Chaos in Medicine, World Scientific: Singapore, 1990.

[5] Emerson, D.R., Cieślicki, K., Gu, X.J. \& Barber, R.W., Biomimetic design of microfluidic manifolds based on a generalised Murray's law, Lab Chip, 6, 447-454, 2006.

[6] Shah, R.K. \& London, A.L., Laminar Flow Forced Convection in Ducts, Academic Press: New York, 1978.

[7] Morini, G.L., Laminar liquid flow through silicon microchannels, J. Fluids Eng., 126, pp. 485-489, 2004.

[8] CFD-ACE+ User Manual: Version 2004, ESI CFD, Huntsville, USA. 